\title{
Impact of insulin resistance on the progression of chronic liver diseases
}

\author{
KOSUKE KAJI, HITOSHI YOSHIJI, MITSUTERU KITADE, YASUHIDE IKENAKA, RYUICHI NOGUCHI, \\ JUNICHI YOSHII, KOJI YANASE, TADASHI NAMISAKI, MASAHARU YAMAZAKI, KEI MORIYA, \\ TATSUHIRO TSUJIMOTO, HIDETO KAWARATANI, TAKEMI AKAHANE, \\ MASAHITO UEMURA and HIROSHI FUKUI
}

Third Department of Internal Medicine, Nara Medical University, Kashihara, Nara, Japan

Received August 13,2008; Accepted September 29, 2008

DOI: $10.3892 /$ ijmm_00000088

\begin{abstract}
Recent studies have revealed a close relationship between insulin resistance (IR) and the progression of chronic liver diseases, although relatively little is known regarding the possible mechanisms involved. The aim of this study was to elucidate the impact of IR on the development of liver fibrosis and hepatocarcinogenesis using obese diabetic Otsuka Long-Evans Tokushima Fatty (OLETF) rats. Liver fibrosis development and glutathione-S-transferase placental form (GST-P)-positive pre-neoplastic lesions were both markedly accelerated in OLETF rats, being induced by pig serum and diethylnitrosamine (DEN), respectively. In the fibrosis experiment, $\alpha$-smooth muscle actin-positive activated hepatic stellate cells (HSCs) also significantly increased in OLETF rats along with augmentation of the hepatic collagen content and transforming growth factor- $\beta_{1}$. Our in vitro study showed that both glucose and insulin stimulated the proliferation of activated HSCs, and the combination treatment exerted an additive effect. In the DEN model, neovascularization, which plays a pivotal role in hepatocarcinogenesis, was up-
\end{abstract}

Correspondence to: Dr Hitoshi Yoshiji, Third Department of Internal Medicine, Nara Medical University, Shijo-cho 840, Kashihara, Nara 634-8522, Japan

E-mail: yoshijih@naramed-u.ac.jp

Abbreviations: CHC, chronic hepatitis C; DEN, diethylnitrosamine; DM, diabetes mellitus; ERK1/2, extracellular signalregulated kinase 1/2; ECs, endothelial cells; GST-P, glutathione-Stransferase placental form; HCC, hepatocellular carcinoma; HSCs, hepatic stellate cells; HUVECs, human umbilical vein endothelial cells; IR, insulin resistance; LETO, Long-Evans Tokushima Otsuka rats; NAFLD, non-alcoholic fatty liver diseases; NASH, nonalcoholic steatohepatitis; OLETF, Otsuka Long-Evans Tokushima Fatty rats; QUICKI, Quantitative Insulin Sensitivity Check Index; $\alpha$-SMA, $\alpha$-smooth muscle actin; TGF, transforming growth factor; VEGF, vascular endothelial growth factor

Key words: hepatocellular carcinoma, angiogenesis, hepatic stellate cells, glucose, vascular endothelial growth factor regulated in OLETF rats almost in parallel with pre-neoplastic lesion development and a potent angiogenic factor, vascular endothelial growth factor. High glucose and insulin also significantly augmented the in vitro neovascularization via extracellular signal-regulated kinase $1 / 2$ phosphorylation. Similar to the effect on the activated HSCs, co-existence of both factors exerted a more potent effect than either single factor. In conclusion, these results indicated that the IR status directly accelerated liver fibrosis development and hepatocarcinogenesis at least partly through the stimulation of activated HSC proliferation and hepatic neovascularization, respectively, in the rat.

\section{Introduction}

Hepatocellular carcinoma (HCC) is a malignancy of worldwide significance, and its prevalence is still increasing in Japan, Western Europe and the US (1). HCC commonly develops in patients with liver cirrhosis. Liver cirrhosis is caused by many etiological factors, such as hepatitis B or C (2). Among them, hepatitis virus $\mathrm{C}(\mathrm{HCV})$ infection is now the most common underlying cause of these clinical entities, i.e., liver cirrhosis and HCC (3). Recent evidence suggests that HCV infection is associated with an increased risk of type 2 diabetes mellitus (DM) (4-6). Cross-sectional human studies showed that insulin resistance (IR), i.e., co-existence of high glucose and insulin, is a consistent finding in patients with type $2 \mathrm{DM}$ (7). Experimental evidence for the contribution of $\mathrm{HCV}$ in the development of IR and DM has been found in the HCV-transgenic mouse model (5). Moreover, several human studies demonstrated that IR is a risk factor of advanced fibrosis in patients with chronic hepatitis $\mathrm{C}$ (CHC), and IR itself may contribute to the progression of liver fibrosis in CHC (8-10). Moreover, a similar close interaction was observed in patients with other morbidities, such as nonalcoholic fatty liver diseases (NAFLD) (11). Either high glucose or insulin increased the production and expression of collagen genes in the activated hepatic stellate cells (HSCs), which play a pivotal role in liver fibrosis development in vitro $(12,13)$.

In addition to liver fibrogenesis, a close interaction between DM and HCC has been documented. DM is associated with a 2- to 3-fold increase in the risk of HCC regardless of the etiology of chronic liver diseases, including 
CHC and non-alcoholic steatohepatitis (NASH) $(14,15)$. It is now recognized that angiogenesis plays an important role in many physiological and pathological events (16). Neovascularization and a potent angiogenic factor; namely, the vascular endothelial growth factor (VEGF), play important roles in hepatocarcinogenesis. It has been reported that VEGF expression and hepatic neovascularization exhibit a stepwise increase in pre-neoplastic lesions during hepatocarcinogenesis (17). Several recent investigations have revealed a close relationship between angiogenesis, high glucose and insulin $(18,19)$. However, the possible interaction between IR, angiogenesis and VEGF during hepatocarcinogenesis has not as yet been elucidated.

In the current study, we examined the in vivo role of IR on the progression of chronic liver diseases, i.e., liver fibrosis development and hepatocarcinogenesis, using obese diabetic Otsuka Long-Evans Tokushima Fatty (OLETF) rats, which develop IR after 10-15 weeks of age and non-insulindependent DM after 25-30 weeks of age (20). We also performed a set of in vitro experiments to elucidate the possible mechanism involved in these processes.

\section{Materials and methods}

Animal treatment. Male OLETF and control Long-Evans Tokushima Otsuka (LETO) rats were generously supplied by Otsuka Pharmaceutical Co. (Tokushima, Japan) (20). At the age of 20 weeks, the LETO and OLETF rats were randomly divided into 8 groups and employed for both fibrosis and hepatocarcinogenesis experiments. In the fibrosis experiment, $1.0 \mathrm{ml} / \mathrm{kg}$ of pig serum (JRH Biosciences, Inc., St. Lenexa, KS, USA) was intraperitoneally injected twice a week for 4 weeks both in the LETO and OLETF rats, which were designated as Group 1 (G1) and G2, respectively. As a negative control, phosphate buffer saline (PBS) was injected instead in the LETO and OLETF rats (G3 and G4, respectively). In the carcinogenesis experiment, LETO (G5) and OLETF (G6) rats received a single injection of $200 \mathrm{mg} / \mathrm{kg}$ of diethylnitrosamine (DEN) (Tokyo Kasei Kogyo Co., Ltd, Tokyo, Japan), partially hepatectomized at week 3 as described previously $(21,22)$, and then sacrificed at the end of week 9. In the carcinogenesis experiment, PBS was injected and partial hepatectomy was performed in the LETO and OLETF rats (G7 and G8, respectively) as a negative control. Each group consisted of 10 rats. At the end of the respective experiment, all rats were anesthetized, the thoracic cavity was opened, and blood samples were withdrawn via cardiac puncture. Several biological markers were assessed by routine laboratory methods. IR was evaluated with the Quantitative Insulin Sensitivity Check Index (QUICKI) as described previously (23). All animal procedures were performed according to standard protocol and in accordance with the standard recommendations for the proper care and use of laboratory animals.

Histological and immunohistochemical staining and quantification. The first section was routinely stained with hematoxylin and eosin for histological examination. Another section was stained with Sirius-red (S-R) to detect fibrosis development. Immunohistochemical staining of enzyme- altered pre-neoplastic lesions; namely, the placental form of glutathione S-transferase (GST-P) (MBL Co., Ltd., Nagoya, Japan) and $\alpha$-smooth muscle actin ( $\alpha$-SMA) (Dako, Kyoto, Japan), using formalin-fixed tissue sections, was performed as described previously $(24,25)$. For determination of neovascularization, we employed immunohistochemical detection of CD31 (BD Biosciences, CA, USA), which is widely used as a marker of neovascularization as previously described with frozen sections (26). Briefly, non-specific antibody binding was blocked with serum diluted at 1:50 in PBS, and the endogenous biotin was blocked with $0.1 \%$ avidin for $15 \mathrm{~min}$, followed by $0.01 \%$ biotin for $15 \mathrm{~min}$. These sections were washed once with PBS after each 15-min incubation period, then reacted with a primary respective antibody for $1 \mathrm{~h}$ at room temperature. After washing twice with PBS, sections were incubated with a second biotin-labeled rabbit anti-rat IgG (Novocastra, CA, USA). The endogenous peroxidase was blocked with $0.3 \%$ hydrogen peroxide in PBS for $30 \mathrm{~min}$, followed by a 5-min washing with PBS. The sections were then incubated with conjugated streptavidin for $30 \mathrm{~min}$, rinsed again in PBS, and finally incubated with diaminobenzidine for $3 \mathrm{~min}$. We did not count the $\alpha$-SMA-positive vessels in the portal area, which were assumed to be hepatic arteries. We only included the $\alpha$-SMA-positive cells in the sinusoidal lining for image analysis. Semi-quantitative analyses of fibrosis development and immunopositive cells of $\alpha$-SMA, GST-P and CD31 of 7 rats were carried out using Adobe Photoshop software and National Institutes of Health image software as described elsewhere (24-26).

Hepatic collagen, transforming growth factor- $\beta_{1}$ and vascular endothelial growth factor expression. After equalization of the protein content, the hepatic collagen content was determined using a Sircol Soluble Collagen Assay Kit (Biocolor Ltd., New Town Abbey, Northern Ireland) in 200 mg of frozen liver samples according to the manufacturer's instructions. TGF- $\beta_{1}$ and VEGF in the liver were determined using an enzymelinked immunosorbent assay (ELISA) kit (R\&D Systems, Tokyo, Japan), according to the manufacturer's instructions.

In vitro proliferation assays of hepatocellular carcinoma. The primary HSCs were isolated from the liver of OLETF rats, as described previously (27) with a minor modification. The cell viability was $>95 \%$ as determined by the Trypan blue exclusion test. Freshly isolated HSCs were plated at a density of $5 \times 10^{5}$ cells $/ \mathrm{ml}$ on uncoated plastic dishes. After a 5-day culture, HSCs became myofibroblast-like with reduced lipid vesicles and increased immunoreactive $\alpha$-SMA, and at 7 days after planting, all cells were well-spread and $\alpha$-SMA positive (28). The effects of D-(+)-glucose (Nacalai Tesque, Kyoto, Japan) and human recombinant insulin (Gibco, Tokyo, Japan) on the proliferation of HSCs were detected by bromodeoxyuridine (BrdU) incorporation using a Cell Proliferation ELISA, BrdU kit (Roche Applied Science, Mannheim, Germany) according to the manufacturer's instructions.

In vitro angiogenesis assay and extracellular signal-regulated kinase 1/2 phosphorylation in endothelial cells (ECs). In vitro angiogenesis was assessed as the formation of capillary-like structures of human umbilical vein endothelial cells 

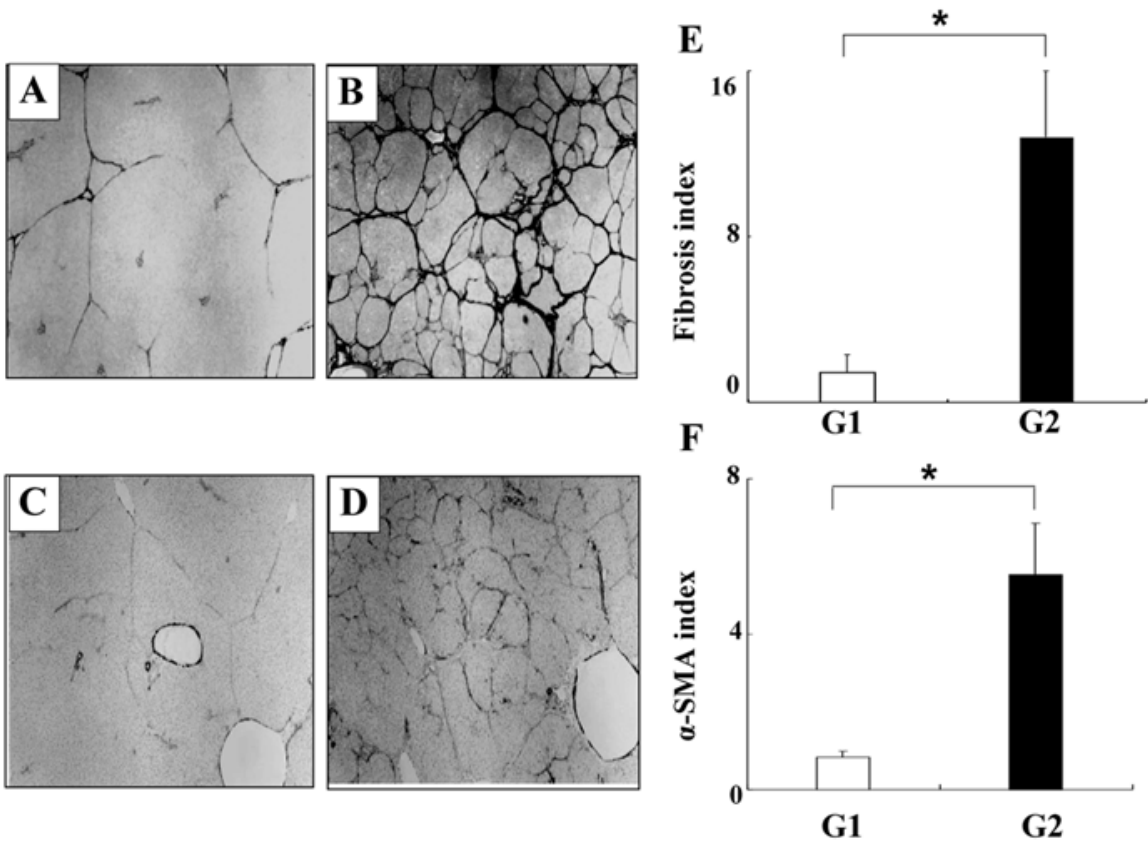

Figure 1. Representative microphotographs of liver fibrosis development and HSC activation. Pig serum resulted in marked fibrosis development in the (B) OLETF G2 rats whereas little fibrosis development was observed in the (A) LETO G1 rats (Sirius-red staining; original magnification, $x 40$ ). Similar to the findings of fibrosis development, the $\alpha$-SMA-immunopositive-activated HSCs significantly increased in the liver of the (D) OLETF G2 rats as compared with the (C) LETO G1 rats (original magnification, $\mathrm{x} 40$ ). (E) Densitometric analysis showed that the fibrosis area almost corresponded to the histological findings. (F) Along with the histological findings, image analysis showed that the $\alpha$-SMA-positive lesions significantly increased in the G2 rats (as compared with the G1 rats) at a similar magnitude to the liver fibrosis development. The data represent the mean $\pm \mathrm{SD}(\mathrm{n}=10)$. Statistically significant difference between the indicated groups $\left({ }^{*} \mathrm{p}<0.01\right)$.

Table I. Characteristic features of the LETO and OLETF rats.

\begin{tabular}{lcc}
\hline Group & LETO & OLETF \\
\hline Body weight (g) & $515.0 \pm 20.8$ & $671.3 \pm 35.2^{\mathrm{a}}$ \\
Liver weight (g) & $14.0 \pm 1.6$ & $25.8 \pm 4.3^{\mathrm{a}}$ \\
Ratio (liver to body) & $0.027 \pm 0.004$ & $0.038 \pm 0.007^{\mathrm{a}}$ \\
Albumin (g/dl) & $3.9 \pm 0.3$ & $3.7 \pm 0.5$ \\
T-Bil (mg/dl) & $0.06 \pm 0.01$ & $0.14 \pm 0.08$ \\
ALT (IU/l) & $37.5 \pm 1.7$ & $98.3 \pm 18.4^{\mathrm{a}}$ \\
Glucose (mg/dl) & $178.5 \pm 17.3$ & $271.3 \pm 41.0^{\mathrm{a}}$ \\
Insulin (nM/ml) & $50.6 \pm 7.1$ & $128.2 \pm 10.4^{\mathrm{a}}$ \\
QUICKI & $0.244 \pm 0.009$ & $0.206 \pm 0.003^{\mathrm{a}}$ \\
\hline
\end{tabular}

Values are the means \pm SD. Statistically different vs. the LETO rats $\left({ }^{\mathrm{a}} \mathrm{P}<0.01\right)$.

(HUVECs) co-cultured with human diploid fibroblasts as described previously (29). The experimental procedure followed the instructions provided with the angiogenesis kit (Kurabo, Tokyo, Japan). Computer-assisted quantitation of tubule formation was performed as in the in vivo assay. The effect of glucose and insulin on extracellular signal-regulated kinase 1/2 (ERK1/2) phosphorylation in HUVECs was evaluated by the Cellular Activation of Signaling ELISA (CASE) kit (ActiveMotif, Tokyo, Japan), according to the manufacturer's protocol.
Statistical analysis. To assess the statistical significance of the inter-group differences in the quantitative data, Bonferroni's multiple comparison test was used after one-way ANOVA. This was followed by Barlett's test to determine the homology of variance.

\section{Results}

General findings in the OLETF and LETO rats. The characteristic features of the OLETF and LETO rats at the age of 20 weeks are shown in Table I. The final body weights and relative liver weights of the OLETF rats were both higher than those of the LETO rats. Regarding the serological data, the serum alanine aminotransferase (ALT) was significantly higher in the OLETF rats than that in the LETO rats, whereas no significant differences in the levels of albumin and total bilirubin were observed. Considering the IR status, the serum levels of glucose and insulin were significantly higher in the OLETF than those in the LETO rats. The value of QUICKI in the OLETF rats was significantly lower than that in the LETO rats, confirming the IR status in the OLETF rats as described previously (20).

Liver fibrosis development in the pig serum-treated rats. Histological examination revealed that 4-week treatment with pig serum resulted in a marked fibrosis development in the OLETF rats (G2) whereas little fibrosis development was observed in the LETO rats (G1) (Fig. 1B and A, respectively). We next carried out the $\alpha$-SMA immunohistochemical analysis to examine the effects of IR status on HSC activation. The 
A

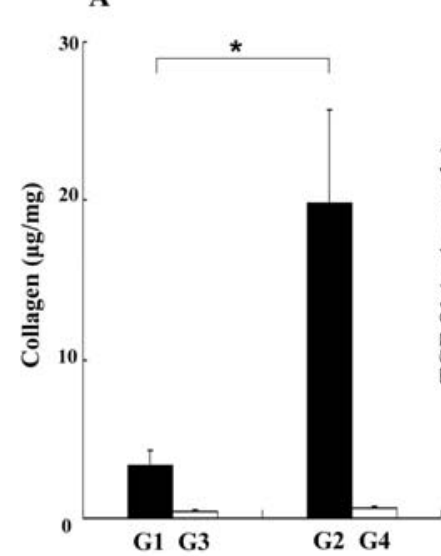

B

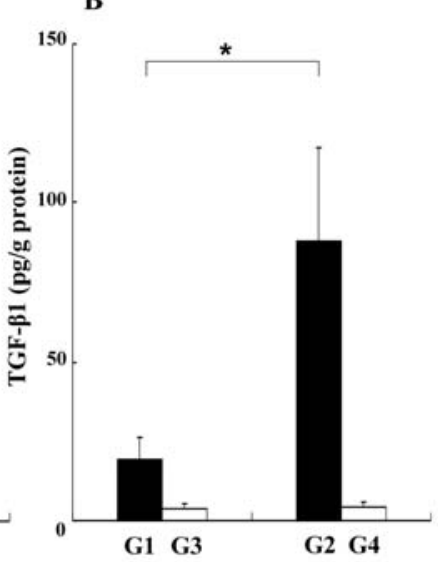

Figure 2. The effect of IR status on the hepatic collagen content (A) and TGF- $\beta_{1}$ expression (B) in the liver of the LETO (G1) and OLETF (G2) rats. Similar to the fibrotic area, hepatic collagen and TGF- $\beta_{1}$ significantly increased in the G2 rats as compared with the G1 rats. The magnitudes of these indices were almost similar to that of $\alpha$-SMA expression. No increase in hepatic collagen and TGF- $\beta_{1}$ was observed in the liver of the PBS-treated LETO and OLETF rats (G3 and G4, respectively). The data represent the mean \pm SD $(n=10)$. Statistically significant difference between the indicated groups $\left({ }^{*} \mathrm{p}<0.01\right)$.

$\alpha$-SMA-immunopositive-activated HSCs were more markedly increased in G2 than in G1 (Fig. 1D and C, respectively). The densitometric analysis confirmed that the activated HSCs increased significantly at a similar magnitude to the liver fibrosis development (Fig. 1E and F, respectively). The hepatic collagen content and TGF- $\beta_{1}$ expression also increased in G2 as compared with G1 (Fig. 2A and B, respectively). No fibrosis development was found in the control groups of the OLETF and LETO rats, which received PBS (G3 and G4, respectively).

Effects of high glucose and insulin on the proliferation of HSCs in vitro. To evaluate whether the IR status, i.e., high glucose and insulin, directly affected the activated HSCs, we examined the effects of high glucose and insulin on the proliferation of the activated HSCs in vitro. We measured BrdU incorporation of HSCs incubated with glucose and insulin at various concentrations $(100,200,400,600 \mathrm{mg} / \mathrm{dl}$ and $1,10,100,1000 \mathrm{nM}$, respectively) for $24 \mathrm{~h}$. As shown in Fig. 3A and B, glucose and insulin treatment stimulated the proliferation of the activated HSCs in a dose-dependent manner. We next examined the combined effect of glucose and insulin on the proliferation of the activated HSCs. Either single treatment with glucose $(271.3 \mathrm{mg} / \mathrm{dl})$ or insulin (128.2 nM), which almost corresponded to the serum levels in the OLETF rats, stimulated the proliferation of the activated HSCs, and co-existence of both conditions exerted a more potent effect as compared with either single condition (Fig. 3C). We also observed that neither serum levels of insulin nor glucose in the OLETF rats promoted proliferation of quiescent primary HSCs (data not shown).

Tumor development in DEN-treated rat liver. In the hepatocarcinogenesis model, the GST-P-positive pre-neoplastic lesions in the OLETF rats (G6) were generally larger than those in the LETO rats (G5) (Fig. 4B and A, respectively). As shown in Fig. 4C, the mean size of the pre-neoplastic lesions in the G6 rats was significantly larger than that in the G5 rats. On the other hand, no marked differences were observed in the number of the GST-P-positive lesions between the G5 and G6 rats (Fig. 4D). No GST-P-positive lesions developed in the PBS-treated OLETF and LETO rats (G7 and G8, respectively) (data not shown).

Effects of high glucose and insulin on neovascularization. Since neovascularization has been shown to play an important
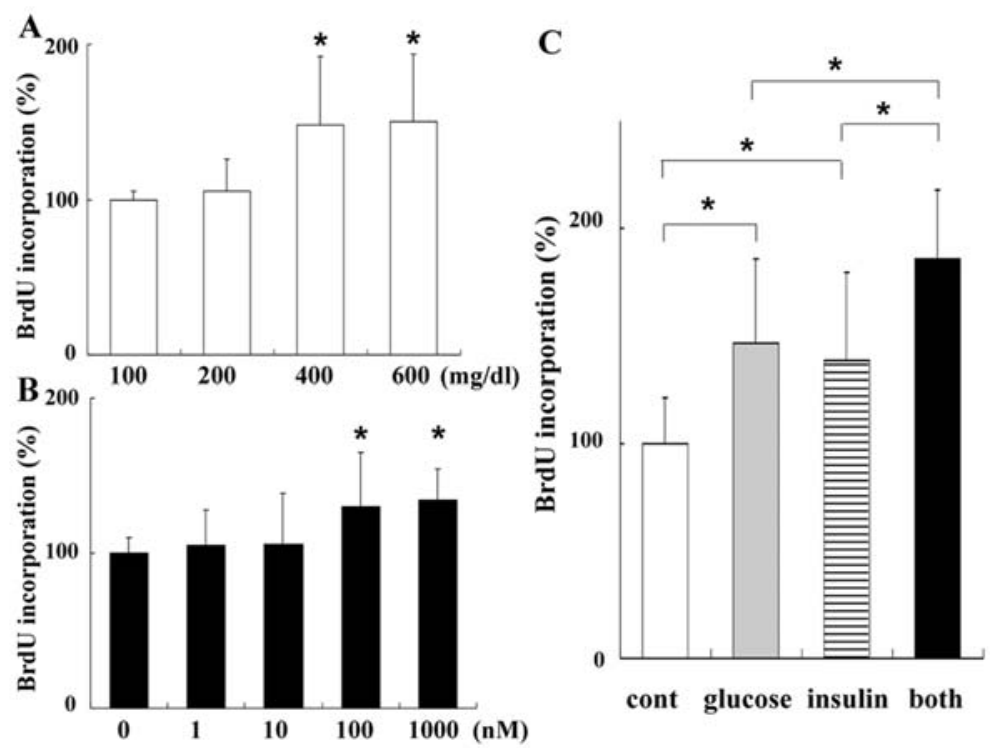

Figure 3. The effects of glucose (A) and insulin (B) on the proliferation of activated HSCs in vitro. Proliferation was measured in the presence of glucose (A) and insulin (B) at various concentrations $(100,200,400,600 \mathrm{mg} / \mathrm{dl}$ and $1,10,100,1000 \mathrm{nM}$, respectively). Both glucose and insulin treatment stimulated the proliferation of activated HSCs in a dose-dependent manner. (C) The effect of co-existence of glucose and insulin on the proliferation of activated HSCs in vitro. The combination treatment of glucose and insulin significantly stimulated the proliferation of activated HSCs as compared with either single treatment. The concentrations of glucose and insulin were $271.3 \mathrm{mg} / \mathrm{dl}$ and $128.2 \mathrm{nM}$, respectively, which almost corresponded to the serum levels in the OLETF rats. The data represent the mean $\pm \mathrm{SD}(\mathrm{n}=12)$. Statistically significant difference between the indicated groups $\left({ }^{*} \mathrm{p}<0.01\right)$. 

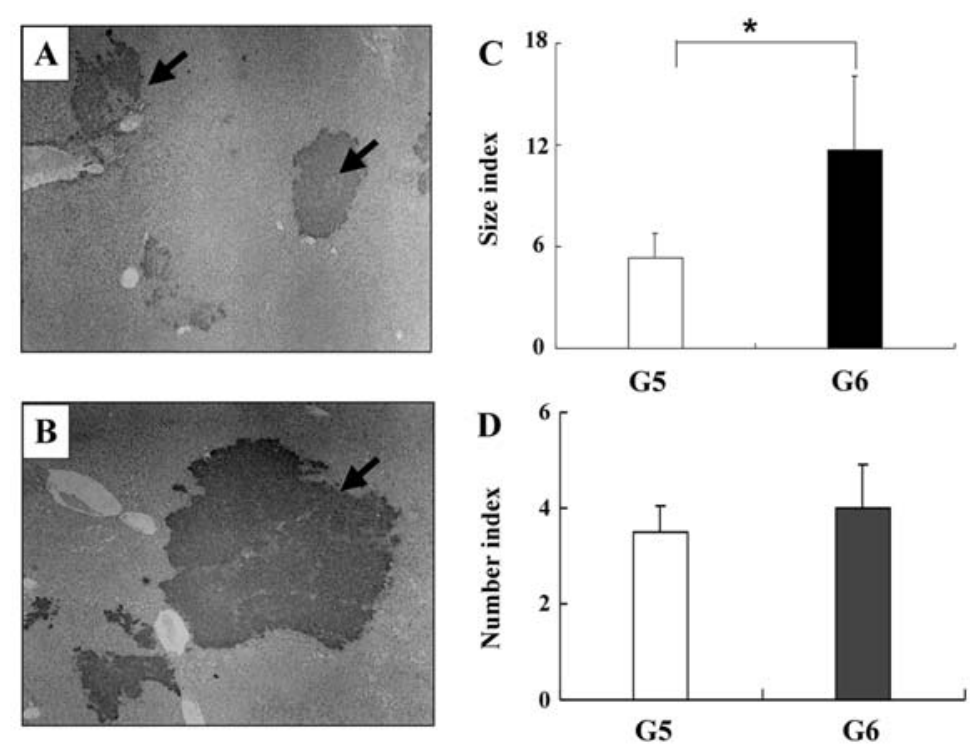

Figure 4. Representative microphotographs and indices of the GST-P-positive pre-neoplastic lesions in the liver. (A) In the DEN-treated LETO (G5) rats, GST-P-positive lesions moderately developed. (B) A marked development of pre-neoplastic lesions was observed in the DEN-treated OLETF (G6) rats as compared with the LETO rats. The arrows indicate the GST-P-positive pre-neoplastic lesions (original magnification, $\mathrm{x} 40$ ). (C and D) The mean size of the pre-neoplastic lesions in the G6 rats was significantly larger than that in the G5 rats, whereas no marked differences were observed in the number of GST-Ppositive lesions between the G5 and G6 rats. The data represent the mean \pm SD $(n=10)$. Statistically significant difference between the indicated groups $(" \mathrm{p}<0.01)$.

A

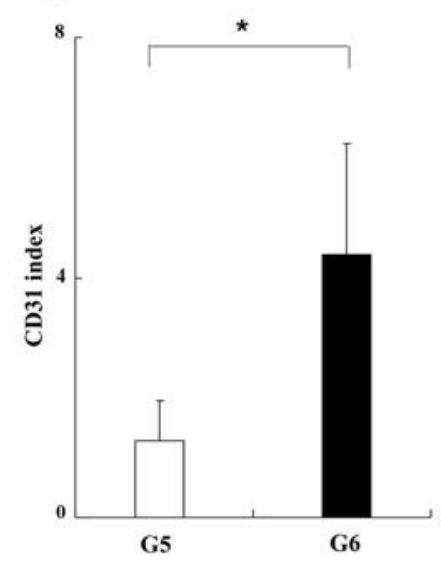

B

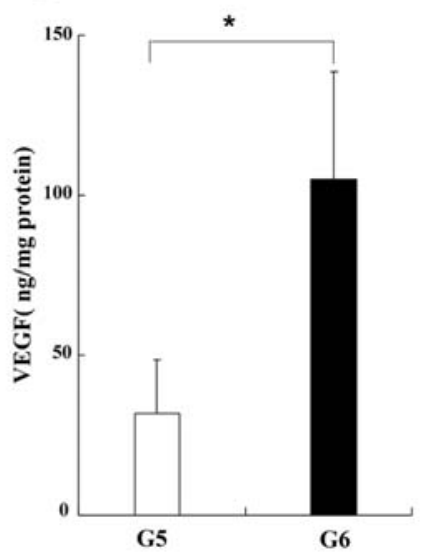

Figure 5. The effects of the IR status on the development of neovascularization and VEGF in the liver. Similar to the development of GSTP-positive lesions, hepatic neovascularization, which was evaluated with immunohistochemical analysis with CD31 (A) and VEGF expression (B) were significantly higher in the OLETF (G6) than in the LETO (G5) rats. The expression levels of neovascularization and VEGF in G6 rats were similar to those during the development of GST-P-positive pre-neoplastic lesions. MV, microvessel. The data represent the mean $\pm \operatorname{SD}(n=10)$. Statistically significant difference between the indicated groups $\left({ }^{*} \mathrm{p}<0.01\right)$.

role in the hepatocarcinogenesis, we investigated the possible involvement of neovascularization in the current study. We first examined the effect of IR on neovascularization in the liver to elucidate whether or not the augmented effect of IR on GST-P-positive lesions is associated with alteration of angiogenesis. We observed that the number of CD31immunopositive neo-vessels was much higher in the liver of the OLETF (G6) than in the LETO (G5) rats (Fig. 5A). These neo-vessels were mainly localized in the pre-neoplastic lesions. We also performed an immunohistochemical analysis with another marker of neovascularization (CD105, endoglin), and the results were similar to those of CD31 (data not shown). Hepatic VEGF expression was also significantly higher in the G6 than in the G5 rats (Fig. 5B). The magnitude of VEGF augmentation was of a similar level to the development of neovascularization.

To elucidate the direct interaction between high glucose, insulin and angiogenesis, we next performed a set of in vitro experiments. Either high glucose or insulin treatment stimulated EC tubular formation, and the combination treatment with glucose and insulin exerted a more potent angiogenic activity as compared with the single condition. These promoting effects were suppressed by treatment with VEGF-neutralizing monoclonal antibody (VEGF-mAb) (Fig. 6). Since high glucose and insulin reportedly stimulate tubular formation of ECs via ERK 1/2 (18), we also examined the effect of glucose and insulin at doses corresponding to the serum levels in the OLETF rats. As shown in Fig. 7, ERK1/2 phosphorylation significantly increased in either glucose- or insulin-treated ECs, and the combination treatment of glucose and insulin exerted more ERK1/2 phosphorylation as compared with either single treatment. Similar to tubular formation, this augmentation was almost attenuated by treatment with VEGF mAb.

\section{Discussion}

Liver cirrhosis and HCC are known as terminal clinical manifestations in chronic liver diseases regardless of the etiology $(1,2,30)$. Recent studies have revealed a possible interaction between these entities and IR; i.e., co-existence of high glucose and insulin. In the current study, we found that 
( I )
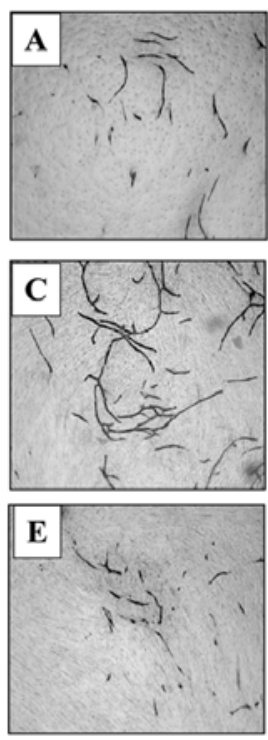

(II)
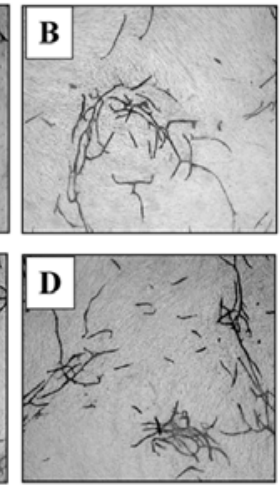

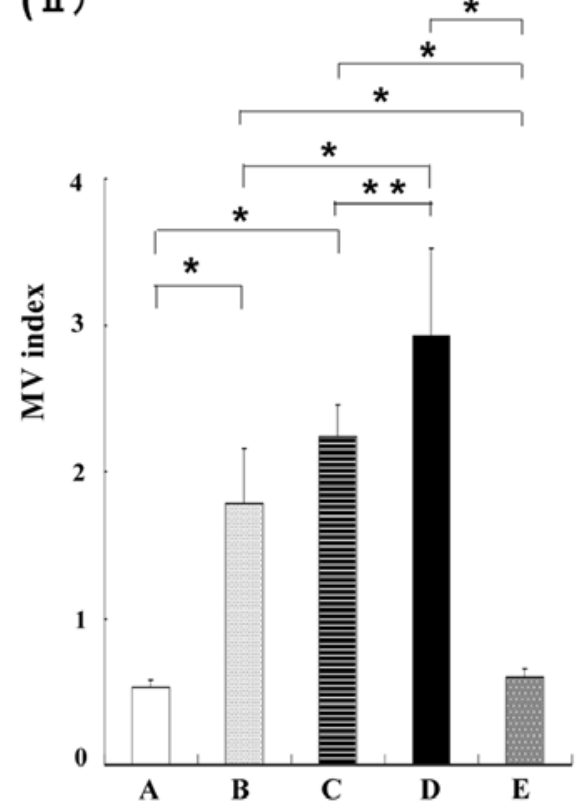

Figure 6. (I) Representative features and index of in vitro EC tubular formation following treatment with glucose and/or insulin. (A) PBS-treated control group. (B and C) Glucose (271.3 mg/dl)- and insulin (128.2 nM)-treated groups, respectively. (D) Combination-treated group. Treatment with either glucose or insulin increased the EC tubule formation as compared with the PBS-treated control group. The combination treatment with glucose and insulin caused a further augmentation as compared with either single agent treatment. (E) These promoting effects were mostly attenuated by treatment with VEGFneutralizing monoclonal antibody (VEGF mAb). (II) Semi-quantitative analysis with image analyzer system confirmed the above-mentioned findings. MV, microvessel. The data are expressed as the mean \pm SD $(n=12)$. Statistically significant differences between the indicated groups $\left({ }^{*} \mathrm{p}<0.01\right.$ and ${ }^{* *} \mathrm{p}<0.05$, respectively).

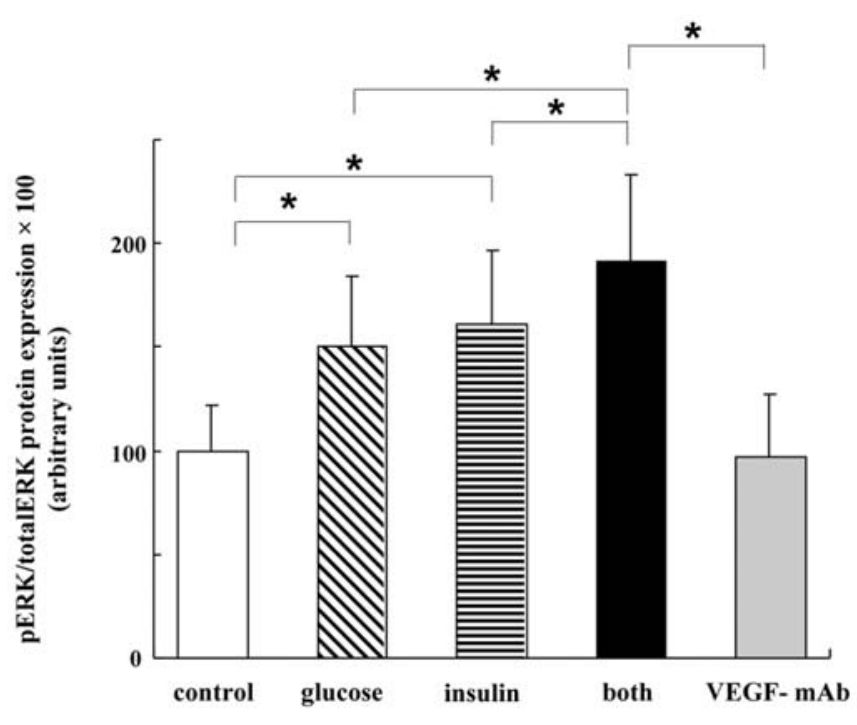

Figure 7. Glucose- and insulin-induced ERK1/2 phosphorylation in the EC. ERK1/2 phosphorylation was significantly increased in either glucose- or insulin-treated EC, and the combination treatment of glucose and insulin exerted more ERK1/2 phosphorylation as compared with either single treatment. Similar to tubular formation, this augmentation was almost attenuated by treatment with VEGF-neutralizing monoclonal antibody (VEGF-mAb). The results are expressed as the ratio of phosphorylated ERK1/2 (pERK) to the total ERK1/2 in HUVEC. The data represent the mean $\pm \mathrm{SD}(\mathrm{n}=8)$. Statistically significant difference between the indicated groups $(* \mathrm{p}<0.01)$.

liver fibrosis development and hepatocarcinogenesis significantly progressed in the presence of IR. It is known that activated HSCs play a central role on the basis of their ability to undergo activation during development of liver fibrosis, and that HSCs produce several important molecules such as TGF- $\beta_{1}(31,32)$. Previous investigations have shown that high glucose and insulin stimulated the proliferation of HSCs $(12,13)$. In this study, we also observed that both glucose and insulin stimulated the proliferation of activated HSCs. Furthermore, we first noticed that the combination treatment with glucose and insulin significantly promoted the proliferation of activated HSCs as compared with either single agent. We did not detect these promoting effects of glucose and insulin on quiescent HSCs. The exact reason of this discrepancy is not clear at this time. It has been reported that insulin receptors were up-regulated during the activation of HSCs (13), and that high glucose stimulated the proliferation at least partly through the NADPH oxidase-mediated reactive oxygen species (ROS), which is known to be associated with the activation of HSCs (12). These phenomena may be involved. Recently, it has been reported that the IR status accelerated the development of steatohepatitis and fibrosis in the NASH experimental model (33). The study showed a close relationship between steatohepatitis and fibrosis through up-regulation of lipogenesis-related genes. In contrast, in the current study, we did not observe steatosis or steatohepatitis in the OLETF and LETO rats. The pig serum model induces liver fibrosis without severe inflammation (34). As we focused mainly on the effect of IR without any additional effect such as inflammation or steatohepatitis-induced cytokines, we employed this model in the present study.

We also observed the promoting effect of IR on the development of pre-neoplastic lesions along with augmentation of intrahepatic neovascularization and VEGF. We previously 
reported that hepatic neovascularization and VEGF exhibited a stepwise increase during hepatocarcinogenesis (17). A recent study suggested that alterations in the hepatic microcirculation occur at a very early stage of liver carcinogenesis (35). As such, we found that neovascularization was augmented mainly in the GST-P-positive pre-neoplastic lesions. Notably, the size of the pre-neoplastic lesions significantly increased under IR whereas the number was not altered. It is possible that the IR status is involved in the so called 'angiogenic switch' rather than in cell transformation itself. It has been reported that both insulin and glucose can induce VEGF expression $(36,37)$. In this study, we found an additive effect of high insulin and glucose on EC tubular formation, and this promoting effect was markedly cancelled by treatment with VEGF mAb. These results indicated that the IR status induced neovascularization at least partly via VEGF. It has been reported that high glucose and insulin stimulate tubular formation of ECs via the ERK1/2 pathway (18). In the current study, we also observed that high glucose and insulin stimulated the ERK1/2 phosphorylation of ECs, and these augmentations were almost attenuated by treatment with VEGF mAb, indicating that the glucose- and insulinmediated ERK1/2 phosphorylation was, at least partly, mediated by VEGF.

Although previous studies conducted to determine the molecular processes associated with fibrosis and angiogenesis were performed independently, recent studies have revealed that both biological phenomena emerge synergistically (38). We and other groups reported that neovascularization significantly increased during liver fibrosis development (38-40). As in the ECs, VEGF has been shown to act on activated HSCs $(39,40)$. Accordingly, it is possible that augmentation of VEGF-mediated neo-vascularization may also play some role in the development of IR-induced liver fibrosis development. Further studies to elucidate the exact mechanism are required in the future.

In conclusion, we demonstrated that the IR status directly accelerated liver fibrosis development and hepatocarcinogenesis via stimulation of activated HSCs and neo-vascularization, respectively. The augmentation of neo-vascularization was associated with VEGF up-regulation. Our in vitro study revealed that high glucose and insulin increased the proliferation of HSCs and EC tubular formation. The coexistence of both factors exerted more potent activities than either single status. These results suggested that an anti-IR therapy may become an alternative new therapeutic strategy against the progression of chronic liver diseases.

\section{References}

1. Schafer DF and Sorrell MF: Hepatocellular carcinoma. Lancet 353: 1253-1257, 1999.

2. Friedman SL: Liver fibrosis - from bench to bedside. J Hepatol 38: S38-S53, 2003.

3. Niederau C, Lange S, Heintges T, et al: Prognosis of chronic hepatitis C: results of a large, prospective cohort study. Hepatology 28: 1687-1695, 1998.

4. Mason AL, Lau JY, Hoang N, et al: Association of diabetes mellitus and chronic hepatitis C virus infection. Hepatology 29: 328-333, 1999.

5. Shintani Y, Fujie H, Miyoshi H, et al: Hepatitis C virus infection and diabetes: direct involvement of the virus in the development of insulin resistance. Gastroenterology 126: 840-848, 2004.
6. Allison ME, Wreghitt T, Palmer CR and Alexander GJ: Evidence for a link between hepatitis $\mathrm{C}$ virus infection and diabetes mellitus in a cirrhotic population. J Hepatol 21: 1135-1139, 1994.

7. Kahn CR: Insulin resistance, insulin insensitivity, and insulin unresponsiveness: a necessary distinction. Metabolism 27: 1893-1902, 1978.

8. Hui JM, Sud A, Farrell GC, et al: Insulin resistance is associated with chronic hepatitis $\mathrm{C}$ virus infection and fibrosis progression. Gastroenterology 125: 1695-1704, 2003.

9. Petrides AS, Vogt C, Schulze-Berge D, Matthews D and Strohmeyer G: Pathogenesis of glucose intolerance and diabetes mellitus in cirrhosis. Hepatology 19: 616-627, 1994.

10. Petit JM, Bour JB, Galland-Jos C, et al: Risk factors for diabetes mellitus and early insulin resistance in chronic hepatitis C. J Hepatol 35: 279-283, 2001.

11. Bugianesi E, McCullough AJ and Marchesini G: Insulin resistance: a metabolic pathway to chronic liver disease. Hepatology 42: 987-1000, 2005.

12. Sugimoto R, Enjoji M, Kohjima M, et al: High glucose stimulates hepatic stellate cells to proliferate and to produce collagen through free radical production and activation of mitogen-activated protein kinase. Liver Int 25: 1018-1026, 2005.

13. Svegliati-Baroni G, Ridolfi F, Di Sario A, et al: Insulin and insulin-like growth factor-1 stimulate proliferation and type I collagen accumulation by human hepatic stellate cells: differential effects on signal transduction pathways. Hepatology 29: 1743-1751, 1999.

14. Davila JA, Morgan RO, Shaib Y, McGlynn KA and El-Serag HB: Diabetes increases the risk of hepatocellular carcinoma in the United States: a population based case control study. Gut 54: 533-539, 2005.

15. El-Serag HB, Tran $T$ and Everhart JE: Diabetes increases the risk of chronic liver disease and hepatocellular carcinoma. Gastroenterology 126: 460-468, 2004.

16. Carmeliet P: Angiogenesis in life, disease and medicine. Nature 438: 932-936, 2005.

17. Yoshiji H, Kuriyama S, Yoshii J, et al: Halting the interaction between vascular endothelial growth factor and its receptors attenuates liver carcinogenesis in mice. Hepatology 39: 1517-1524, 2004.

18. Shigematsu S, Yamauchi K, Nakajima K, Iijima S, Aizawa T and Hashizume K: D-glucose and insulin stimulate migration and tubular formation of human endothelial cells in vitro. Am J Physiol 277: E433-E438, 1999.

19. Nakao-Hayashi J, Ito H, Kanayasu T, Morita I and Murota S: Stimulatory effects of insulin and insulin-like growth factor I on migration and tube formation by vascular endothelial cells. Atherosclerosis 92: 141-149, 1992.

20. Sato T, Asahi Y, Toide K and Nakayama N: Insulin resistance in skeletal muscle of the male Otsuka Long-Evans Tokushima Fatty rat, a new model of NIDDM. Diabetologia 38: 1033-1041, 1995.

21. Solt DB, Medline A and Farber E: Rapid emergence of carcinogen-induced hyperplastic lesions in a new model for the sequential analysis of liver carcinogenesis. Am J Pathol 88: 595-618, 1977.

22. Tamura K, Nakae D, Horiguchi K, et al: Inhibition by N-(4hydroxyphenyl)retinamide and all-trans-retinoic acid of exogenous and endogenous development of putative preneoplastic, glutathione S-transferase placental form-positive lesions in the livers of rats. Carcinogenesis 18: 2133-2141, 1997.

23. Katz A, Nambi SS, Mather K, et al: Quantitative insulin sensitivity check index: a simple, accurate method for assessing insulin sensitivity in humans. J Clin Endocrinol Metab 85: 2402-2410, 2000.

24. Obara T, Makino T, Ura H, et al: Comparative histochemical investigation of the glutathione S-transferase placental form and gamma-glutamyltranspeptidase during $\mathrm{N}$-nitrosobis(2-hydroxypropyl)amine-induced pancreatic carcinogenesis in hamsters. Carcinogenesis 7: 801-805, 1986.

25. Yoshiji H, Kuriyama S, Yoshii J, et al: Angiotensin-II type 1 receptor interaction is a major regulator for liver fibrosis development in rats. Hepatology 34: 745-750, 2001.

26. Yoshiji H, Kuriyama S, Hicklin DJ, et al: KDR/Flk-1 is a major regulator of vascular endothelial growth factor-induced tumor development and angiogenesis in murine hepatocellular carcinoma cells. Hepatology 30: 1179-1186, 1999. 
27. Horie T, Sakaida I, Yokoya F, Nakajo M, Sonaka I and Okita K: L-cysteine administration prevents liver fibrosis by suppressing hepatic stellate cell proliferation and activation. Biochem Biophys Res Commun 305: 94-100, 2003.

28. Shimizu I, Ma YR, Mizobuchi Y, et al: Effects of Sho-saiko-to, a Japanese herbal medicine, on hepatic fibrosis in rats. Hepatology 29: 149-160, 1999.

29. Yoshiji H, Kuriyama S, Noguchi R, et al: Combination of vitamin $\mathrm{K} 2$ and the angiotensin-converting enzyme inhibitor, perindopril, attenuates the liver enzyme-altered preneoplastic lesions in rats via angiogenesis suppression. J Hepatol 42: 687-693, 2005.

30. Sakaida I, Hironaka K, Uchida K, Suzuki C, Kayano K and Okita K: Fibrosis accelerates the development of enzymealtered lesions in the rat liver. Hepatology 28: 1247-1252, 1998.

31. Pinzani $\mathrm{M}$ and Marra F: Cytokine receptors and signaling in hepatic stellate cells. Semin Liver Dis 21: 397-416, 2001.

32. Friedman SL: Cytokines and fibrogenesis. Semin Liver Dis 19: 129-140, 1999.

33. Ota $\mathrm{T}$, Takamura $\mathrm{T}$, Kurita $\mathrm{S}$, et al: Insulin resistance accelerates a dietary rat model of nonalcoholic steatohepatitis. Gastroenterology 132: 282-293, 2007.
34. Bhunchet E, Eishi Y and Wake K: Contribution of immune response to the hepatic fibrosis induced by porcine serum. Hepatology 23: 811-817, 1996.

35. Frachon S, Gouysse G, Dumortier J, et al: Endothelial cell marker expression in dysplastic lesions of the liver: an immunohistochemical study. J Hepatol 34: 850-857, 2001.

36. Natarajan R, Bai W, Lanting L, Gonzales $\mathrm{N}$ and Nadler J: Effects of high glucose on vascular endothelial growth factor expression in vascular smooth muscle cells. Am J Physiol 273: H2224-H2231, 1997.

37. Lu M, Amano S, Miyamoto K, et al: Insulin-induced vascular endothelial growth factor expression in retina. Invest Ophthalmol Vis Sci 40: 3281-3286, 1999.

38. Kalluri R and Sukhatme VP: Fibrosis and angiogenesis. Curr Opin Nephrol Hypertens 9: 413-418, 2000.

39. Yoshiji H, Kuriyama S, Yoshii J, et al: Vascular endothelial growth factor and receptor interaction is a prerequisite for murine hepatic fibrogenesis. Gut 52: 1347-1354, 2003.

40. Ankoma-Sey V, Wang Y and Dai Z: Hypoxic stimulation of vascular endothelial growth factor expression in activated rat hepatic stellate cells. Hepatology 31: 141-148, 2000. 